\title{
Strongly Semicontinuous Domains and Semi-FS Domains
}

\author{
Qingyu He and Luoshan Xu \\ Department of Mathematics, Yangzhou University, Yangzhou 225002, China \\ Correspondence should be addressed to Luoshan Xu; luoshanxu@hotmail.com
}

Received 15 May 2014; Revised 10 July 2014; Accepted 18 July 2014; Published 17 August 2014

Academic Editor: Hee S. Kim

Copyright (C) 2014 Q. He and L. Xu. This is an open access article distributed under the Creative Commons Attribution License, which permits unrestricted use, distribution, and reproduction in any medium, provided the original work is properly cited.

\begin{abstract}
We are mainly concerned with some special kinds of semicontinuous domains and relationships between them. New concepts of strongly semicontinuous domains, meet semicontinuous domains and semi-FS domains are introduced. It is shown that a dcpo $L$ is strongly semicontinuous if and only if $L$ is semicontinuous and meet semicontinuous. It is proved that semi-FS domains are strongly semicontinuous. Some interpolation properties of semiway-below relations in (strongly) semicontinuous bc-domains are given. In terms of these properties, it is proved that strongly semicontinuous bc-domains, in particular strongly semicontinuous lattices, are all semi-FS domains.
\end{abstract}

\section{Introduction}

The theory of continuous lattices [1] and the more general theory of domains [2] initiated by Scott provide a mathematical foundation for the denotational semantics of programming languages and are closely linked to theoretical computer science, general topology and logics [3-5]. The purpose of the theory of domains is to give models for spaces to define computable functions. The idea is that the semantics of a programming language should be formally specified in terms of a small number of basic mathematical constructions on partial orders of information. Intuitively, we say that a state $x$ approximates a state $y$ if any computation of $y$ yields the information of $x$ at some finite stage. A logic-oriented approach to domain theory to formalize the properties of computation is provided in $[2,6]$.

So far, continuous lattices were generalized to other types of order structures, such as quasicontinuous posets $[7,8]$, $Z$-continuous posets [9], and $Z$-precontinuous posets [10]. Motivated by the concept of semiprime ideals studied by Rav in [11], Zhao in [12] first introduced the concept of semicontinuous lattices and showed that semicontinuous lattices have many properties similar to that of continuous lattices. Li and $\mathrm{Wu}$ [13] studied properties of semicontinuous lattices. Bi and $\mathrm{Xu}$ [14] introduced the semi-Scott topology and the semiLawson topology on semicontinuous lattices. Jiang and Shi [15] discussed characterizations of pseudoprimes and studied strong retracts of (stable) semicontinuous lattices. In [16], Li introduced semiprime sets and generalized semicontinuous lattices to semicontinuous domains.

Note that, in the definition of a semicontinuous lattice $L$, the condition used is that, for every element $x \in L, \vee \Downarrow x \geq x$. This condition is too weak to guarantee that every element can be approximated by elements which are below and semiway-below it in a semicontinuous lattice. To guarantee the mentioned approximation property in a suitable class of semicontinuous lattices, or even of semicontinuous domains, we in this paper introduce the concept of strongly semicontinuous domains. It turns out that strongly semicontinuous domains have domain-like features that every element can be approximated by elements below and semiway-below it. The concept of meet semicontinuous domains is also introduced. It is obtained that a dcpo is strongly semicontinuous if and only if it is semicontinuous and meet semicontinuous. Moreover, inspired by the definition of FS-domains posed by Jung [17] in the realm of domains, we introduce the concept of semi-FS domains which are defined by the existence of some approximating identities consisting of finitely separated functions in the realm of dcpos. It is proved that every semi-FS domain is a strongly semicontinuous domain and that every strongly semicontinuous bc-domain, in particular every strongly semicontinuous lattice, is a semi-FS domain. A counterexample is constructed to show that a strongly semicontinuous domain need not be a semi-FS domain. 
We organize the paper as follows: Section 2 gives preliminaries; Section 3 investigates strongly semicontinuous domains; Section 4 introduces semi-FS domains and discusses their properties.

\section{Preliminaries}

We give some basic concepts and results which will be used in the sequel. Most of them come from [4, 12]. For other unstated concepts please refer to [16].

A subset $D$ of a poset $L$ is called directed (resp., filtered) if it is nonempty and every finite subset of $D$ has an upper (resp., a lower) bound in $D$. A poset in which every directed set has a supremum is called a $d c p o$. For a subset $A$ of a poset $L$, let $\downarrow A=\{y \in L: \exists x \in A, y \leq x\}$ and $\uparrow A=\{y \in L: \exists x \in A, y \geq$ $x\}$. We say that $A$ is a lower set (resp., an upper set) if $\downarrow A=A$ (resp., $\uparrow A=A$ ). A subset of $L$ is an ideal (resp., a filter) if and only if it is a directed lower set (resp., filtered upper set). A principal ideal (resp., principal filter) is a set of the form $\downarrow x=\{y \in L: y \leq x\}$ (resp., $\uparrow x=\{y \in L: x \leq y\}$ ). The set of all ideals (resp. filters) in $L$ is denoted by $I d(L)$ (resp., Filt $(L)$ ). For an ideal $P \in I d(L), P$ is said to be prime if $L \backslash P=\emptyset$ or $L \backslash P \in \operatorname{Filt}(L)$. We denote the family of all prime ideals of $L$ by $P I(L)$. A poset is called bounded complete, if every subset that is bounded above has a supremum. A bounded complete dcpo is called a $b c-d c p o$.

Let $S \subseteq L$. We denote $S \downarrow=\{y \in L: \forall x \in S, y \leq x\}$ and $S \uparrow=\{y \in L: \forall x \in S, y \geq x\}$ the set of upper bounds and lower bounds of $S$, respectively.

Lemma 1 (see [16, Lemma 3.2.8]). Let $L$ be a poset, $A, B \subseteq L$. If $A \subseteq B$, then we have

$$
A \uparrow \downarrow \subseteq B \uparrow \downarrow, \quad A \downarrow \uparrow \subseteq B \downarrow \uparrow .
$$

Definition 2 (see [11]). Let $L$ be a lattice. An ideal $I$ of $L$ is said to be semiprime if for any $x, y, z \in L, x \wedge y \in I$ and $x \wedge z \in I$ imply $x \wedge(y \vee z) \in I$.

Lemma 3 (see [12, Lemma 1.2]). An ideal $P$ of a lattice $L$ is semiprime if and only if there exist prime ideals $P_{i}(i \in I)$ of $L$ such that $P=\bigcap_{i \in I} P_{i}$.

Thus every prime ideal is semiprime, and $P I(L) \subseteq R d(L)$. And by Lemma 3, we can generalize the concept of semiprime ideals to the setting of posets.

Definition 4. Let $L$ be a poset and $S \subseteq L$ an ideal of $L$. If there is a family of prime ideals $\left\{P_{i}\right\}_{i \in I}$ such that $S=\bigcap_{i \in I} P_{i}$, then $S$ is called a semiprime ideal. The family of all semiprime ideals of $L$ is denoted by $\operatorname{Rd}(L)$.

$\mathrm{Li}$ in [16] generalized semiprime ideals to semiprime sets.

Definition 5 (see [16]). Let $L$ be a poset. A set $S \subseteq L$ is said to be semiprime if there exists a family of prime ideals $P_{i}(i \in I)$ of $L$ such that $S=\bigcap_{i \in I} P_{i}$.

We denote the family of all semiprime sets of $L$ with $S P(L)$. Clearly, a semiprime set need not to be directed. If a semiprime set $S$ is directed, then $S$ is a semiprime ideal. For a dcpo, we have the following relation:

$$
P I(L) \subseteq R d(L)=S P(L) \cap I d l(L) \subseteq S P(L) .
$$

Proposition 6. Let $L$ be a bc-dcpo. Then $S P(L)=R d(L)$.

Proof. It suffices to show that $S P(L) \subseteq R d(L)$. Let $A \in S P(L)$. Then by Definition 5 there exists a family of prime ideals $P_{i}(i \in I)$ of $L$ such that $A=\bigcap_{i \in I} P_{i}$. For any $y_{1}, y_{2} \in A$, we have $y_{1}, y_{2} \in P_{i}$ for all $i \in I$. Since $P_{i}$ is directed, there exists $y \in P_{i}$ such that $y_{1}, y_{2} \leq y$. As $L$ is a bc-dcpo, we see that $y_{1} \vee y_{2}$ exists and $y_{1} \vee y_{2} \in P_{i}$ for all $i \in I$. Therefore, $y_{1} \vee y_{2} \in A$. So, $A$ is directed and $A \in R d(L)$, as desired.

Lemma 7. For a $b c$-dcpo $L$, let $M \subseteq L$ and $L^{\top}=L \cup\{\top\}$ be the complete lattice obtained from $L$ by adjoining a top element $T$. Then we have

(i) $M \in P I(L)$ if and only if $M \in P I\left(L^{\top}\right)$;

(ii) $M \in R d(L)$ if and only if $M \in R d\left(L^{\top}\right)$.

Proof. (i) Suppose that $M \in P I\left(L^{\top}\right)$. Since $M \subseteq L$, we have $L \backslash M=L^{\top} \backslash M \in \operatorname{Filt}(L)$ and $M \in P I(L)$.

Conversely, let $M \in P I(L)$. Then $M \in I d(L)$ and $L \backslash M \in$ Filt $(L)$. So, $L^{\top} \backslash M=(L \backslash M) \cup\{\top\} \in \operatorname{Filt}\left(L^{\top}\right)$ and $M \in P I\left(L^{\top}\right)$.

(ii) Suppose that $M \in R d(L)$. Then by Definition $4, M=$ $\bigcap_{i \in I} P_{i}$, where $P_{i} \in P I(L)(i \in I)$. It follows from (i) above that $P_{i} \in P I\left(L^{\top}\right)$ for all $i \in I$; thus, $M \in R d\left(L^{\top}\right)$.

Conversely, let $M \in R d\left(L^{\top}\right)$. By Lemma 3, there exist prime ideals $P_{i}(i \in I)$ of $L^{\top}$ such that $M=\bigcap_{i \in I} P_{i}$. Since $\top \notin M, \top \notin P_{i_{0}}$ for some $i_{0} \in I$. Let $J=\left\{j \in I: \top \notin P_{j}\right\}$. Then for any $i \in I \backslash J$, we see that $\mathrm{\top} \in P_{i}=L^{\top}$. By (i) above, we have $P_{j} \in P I(L)$ for each $j \in J$. Then $M=\bigcap_{j \in J} P_{j}$, and $M \in S P(L)$. By Proposition 6, $M \in R d(L)$.

In a poset $L$, we say that $x$ is way-below $y$, or $x$ approximates $y$, written $x \ll y$, and if $D$ is directed with existing $\sup D$ and $\sup D \geq y$, then $x \leq d$ for some $d \in D$. Equivalently, $x \ll y$ iff $x \in I$ for every ideal $I$ of $L$ such that $y \leq \sup I$ whenever sup $I$ exists. We use $\downarrow x$ to denote the set $\{a \in L: a \ll x\}$. If $L$ is a dcpo and, for every element $x \in L$, the set $\downarrow x$ is directed and $\sup \downarrow x=x$, then $L$ is called a domain. A domain $L$ is called an $L$-domain if for each $x \in L$, the principal ideal $\downarrow x$ is a complete lattice. A complete lattice which is a domain is called a continuous lattice.

For complete lattices, replacing ideals with semiprime ideals, Zhao in [12] defined a weak form of the way-below relation.

Definition 8 (see [12]). Let $L$ be a complete lattice. Define the semiway-below relation $\Leftarrow$ on $L$ as follows: for $x, y \in L, x \Leftarrow$ $y$ if for any semiprime ideal $I$ of $L, y \leq \vee I$ implies $x \in I$. For each $x \in L$, we write $\Downarrow x=\{y \in L: y \Leftarrow x\}, \Uparrow x=\{y \in L$ : $x \Leftarrow y\}$.

Definition 9 (see [12]). A complete lattice $L$ is said to be semicontinuous, if for any $x \in L, x \leq \mathrm{V}(\Downarrow x)$.

Zhao [12] showed that the interpolation property holds in semicontinuous lattices. 
Theorem 10 (see [12, Theorem 1.8]). If L is a semicontinuous lattice, then $x \Leftarrow y$ implies the existence of a $z \in L$ such that $x \Leftarrow z \Leftarrow y$.

\section{Strongly Semicontinuous Domains}

In terms of semiprime sets, semicontinuous lattices can be generalized to semicontinuous domains. And then strongly semicontinuous domains will be defined.

Definition 11. Let $L$ be a poset. Define the relation $\Leftarrow$ on $L$ as follows: for any $x, y \in L, y \Leftarrow x$ if for any semiprime set $S$ of $L, x \in S \uparrow \downarrow$ implies $y \in S$. An element $k$ of $L$ is said to be semicompact if $k \Leftarrow k$. For each $x \in L$, we write $\Downarrow x=\{y \in$ $L: y \Leftarrow x\}$ and $\Uparrow x=\{y \in L: x \Leftarrow y\}$.

Proposition 12. Let $L$ be a dcpo; then,

$$
\begin{aligned}
\Downarrow x & =\cap\{S \in S P(L): x \in S \uparrow \downarrow\}=\cap\{S \in R d(L): x \leq \vee S\} \\
& =\cap\{P \in P I(L): x \leq \vee P\} .
\end{aligned}
$$

So, for each $x \in L, \Downarrow x \in S P(L)$. If $L$ is a bc-dcpo, then $\Downarrow x \in$ $R d(L)$.

Proof. Note that $J \uparrow \downarrow=\downarrow(\vee J)$ for each $J \in I d(L)$. So, by Lemma 1 , we have that

$$
\begin{aligned}
\Downarrow & x \\
& =\cap\{S \in S P(L): x \in S \uparrow \downarrow\} \subseteq \cap\{S \in R d(L): x \in \downarrow(\vee S)\} \\
& \subseteq \cap\{P \in P I(L): x \in \downarrow(\vee P)\} .
\end{aligned}
$$

Let $A=\cap\{P \in P I(L): x \in \downarrow(\vee P)\}$. Then $A \in S P(L)$.

Next we show that $A \subseteq \Downarrow x$. Let $y \in A$. For any $M \in S P(L)$ with $x \in M \uparrow \downarrow$, by Definition 5 there exists a family of prime ideals $\left\{P_{i}\right\}_{i \in I}$ such that $M=\bigcap_{i \in I} P_{i}$. It follows from Lemma 1 that $x \in M \uparrow \downarrow \subseteq P_{i} \uparrow \downarrow=\downarrow\left(\vee P_{i}\right)$ for each $i \in I$. Since $y \in A=$ $\cap\{P \in P I(L): x \in \downarrow(\vee P)\}$, we have that $y \in P_{i}$ for each $i \in I$ and $y \in \bigcap_{i \in I} P_{i}=M$. By Definition 11, $y \Leftarrow x$. So, $A \subseteq \Downarrow x$, as desired.

In [16], the semiway-below relation $\Leftarrow_{s}$ on a poset was defined [16, Definition 3.2.2] in a different way. For a dcpo $L$ and $x \in L$, it is established in [16, Proposition 3.2.5] that $\Downarrow_{s} x=\cap\{P \in P I(L): x \leq \vee P\}$. So, by Proposition 12 above, we see that $\Downarrow x=\Downarrow_{s} x$. This means that for a dcpo $L$ and $x, y \in L, y \Leftarrow{ }_{s} x \Leftrightarrow y \Leftarrow x$. Thus, in the setting of dcpos, Definition 11 is equivalent to Definition 3.2.2 in [16].

Note that for a poset $L$ and $x, y \in L, y \Leftarrow_{s} x$ need not imply $y \Leftarrow x$.

Remark 13. Let $L$ be a dcpo and $a, b, c, d \in L$. Then it is easy to check that

(1) $a \Leftarrow b$ does not imply $a \leq b$, the typical modular lattice $M_{5}$ is a counterexample;

(2) if $a \leq b \Leftarrow c \leq d$, then $a \Leftarrow d$. If $a \Leftarrow b \Leftarrow c$, then $a \Leftarrow c$;
(3) if $a \vee b$ exists and $a, b \Leftarrow c$, then $a \vee b \Leftarrow c$;

(4) for any $a, b \in L, a \ll b$ implies $a \Leftarrow b$.

Definition 14 (see [16]). A dcpo $L$ is called a semicontinuous dcpo if for all $x \in L, x \in(\Downarrow x) \uparrow \downarrow$. A semicontinuous dcpo will also be called a semicontinuous domain. A bc-dcpo which is semicontinuous will be called a semicontinuous bc-domain.

By Proposition 12, one can immediately have the following.

Proposition 15. Let $L$ be a dcpo. Then $L$ is semicontinuous iff for any $x \in L, \Downarrow x$ is the smallest semiprime set $S$ such that $x \in S \uparrow \downarrow$.

Proposition 16. Let $L$ be a dcpo. If, for any $x \in L$, there exists a subset $A \subseteq \Downarrow x$ and $x \in A \uparrow \downarrow$, then $L$ is semicontinuous.

Proof. It follows from Lemma 1 and Definition 14.

Strengthening the condition in Definition 14, we give the following.

Definition 17. A dcpo $L$ is said to be strongly semicontinuous if for each $x \in L$,

$$
x \in(\Downarrow x \cap \downarrow x) \uparrow \downarrow
$$

A dcpo (bc-dcpo) which is strongly semicontinuous will be called a strongly semicontinuous domain (strongly semicontinuous bc-domain). A complete lattice which is strongly semicontinuous will be called a strongly semicontinuous lattice.

Clearly, every strongly semicontinuous domain is semicontinuous, a semicontinuous domain $L$ satisfying the condition $x \in(\Downarrow x \cap \downarrow x) \uparrow \downarrow(\forall x \in L)$ is strongly semicontinuous. It is easy to see that, for a dcpo $L$ without proper prime ideals, every pair of elements in $L$ has the semiway-below relation $\Leftarrow$ and $L$ is strongly semicontinuous. However, a semicontinuous domain need not be strongly semicontinuous. The following counterexample first appeared in [18].

Example 18 (see [18]). Let $L=\{\perp, a, b, x, \top\} \cup\left\{x_{n}: n=\right.$ $0,1, \ldots\}$. The partial order on $L$ is defined by $\perp \leq a, b \leq x_{0} \leq$ $\cdots \leq x_{n} \cdots \leq \mathrm{T}, \perp \leq x \leq \mathrm{T}$. It is clear that the prime ideals of $L$ are $L \backslash \uparrow x$ and $L$. We observe that, for any $t \in L, \Downarrow t=$ $L \backslash \uparrow x$ and $t \leq \vee \Downarrow t$. Thus, $L$ is a semicontinuous domain. However, note that, in this example $x \notin(\Downarrow x \cap \downarrow x) \uparrow \downarrow$, which yields that $L$ is not strongly semicontinuous.

Proposition 19. Every domain is a strongly semicontinuous domain.

Proof. It follows from Remark 13(4) that $\forall x \in L$, $\downarrow x \subseteq \Downarrow x \cap \downarrow x$. By Lemma $1, x \in \downarrow x=\downarrow(\vee \downarrow x)=(\downarrow x) \uparrow \downarrow \subseteq$ $(\Downarrow x \cap \downarrow x) \uparrow \downarrow$. Thus, $L$ is a strongly semicontinuous. 
$\mathrm{Wu}$ and $\mathrm{Li}$ in [19] introduced the following concept of meet semicontinuous lattices.

Definition 20 (see [19]). A complete lattice $L$ is said to be meet semicontinuous if for any $x \in L$ and $I \in R d(L), x \wedge(\vee I)=$ $\vee(x \wedge I)$.

It is known that a semicontinuous lattice need not be a meet semicontinuous lattice. However, it is proved in [18] that strongly semicontinuous lattices are all meet semicontinuous. Generalize meet semicontinuous lattices, we have the following

Definition 21. A dcpo $L$ is said to be meet semicontinuous if for any $x \in L$ and $S \in S P(L),(\downarrow x \cap S) \uparrow \downarrow=\downarrow x \cap S \uparrow \downarrow$.

It is easy to check that, for complete lattices, the meet semicontinuity in Definitions 20 and 21 are equivalent.

Proposition 22. Every strongly semicontinuous domain is meet semicontinuous.

Proof. Suppose that $L$ is a strongly semicontinuous domain. For any $x \in L$ and $S \in S P(L)$, by Lemma 1 , it is easy to see that $\downarrow x \cap S \uparrow \downarrow \supseteq(\downarrow x \cap S) \uparrow \downarrow$. So, it suffices to show that $\downarrow x \cap S \uparrow \downarrow \subseteq$ $(\downarrow x \cap S) \uparrow \downarrow$. To this end, let $t \in \downarrow x \cap S \uparrow \downarrow$. Then $t \in S \uparrow \downarrow$ and $t \in(\Downarrow t \cap \downarrow t) \uparrow \downarrow$ since $L$ is strongly semicontinuous. For any $r \in \Downarrow t \cap \downarrow t$, we have that $r \Leftarrow t \in S \uparrow \downarrow$ and $r \leq t \leq x$. Thus, by Definition 11, $r \in S$ and $r \in \downarrow x \cap S$. By the arbitrariness of $r \in \Downarrow t \cap \downarrow t$, we see that $\Downarrow t \cap \downarrow t \subseteq \downarrow x \cap S$. Therefore, $t \in(\Downarrow t \cap \downarrow t) \uparrow \downarrow \subseteq(\downarrow x \cap S) \uparrow \downarrow$. So, $\downarrow x \cap S \uparrow \downarrow \subseteq(\downarrow x \cap S) \uparrow \downarrow$, as desired.

Remark 23. Note that, for the complete lattice in Example 18 and the prime ideal $L \backslash \uparrow x$, we have $\downarrow x \cap(L \backslash \uparrow x) \uparrow \downarrow \neq(\downarrow x \cap$ $(L \backslash \uparrow x)) \uparrow \downarrow$, revealing that $L$ is not meet semicontinuous.

Theorem 24. A dcpo $L$ is strongly semicontinuous iff $L$ is semicontinuous and meet semicontinuous.

Proof. $\Rightarrow$ : By Proposition 22.

$\Leftarrow$ : For each $x \in L$, by the semicontinuity of $L$ we have that $x \in(\Downarrow x) \uparrow \downarrow$. It follows from meet semicontinuity of $L$ that $x \in \downarrow x \cap(\Downarrow x) \uparrow \downarrow=(\downarrow x \cap \Downarrow x) \uparrow \downarrow$. So, by Definition 17, $L$ is strongly semicontinuous.

Proposition 25. For a (strongly) semicontinuous bc-domain $L$, let $L^{\top}=L \cup\{T\}$ be the complete lattice obtained from $L$ by adjoining a top element $\mathrm{T}$. Then $L^{\top}$ is a (strongly) semicontinuous lattice.

Proof. Firstly, we show that $\Downarrow_{L^{\top}} x \supseteq \Downarrow_{L} x$ for each $x \in L$. Let $y \Leftarrow_{L} x$. Then for any $P \in R d\left(L^{\top}\right)$ with $\vee_{L^{\top}} P \geq x$, if $P=L^{\top}$, then $y \in P$. If $P \neq L^{\top}$, then $T \notin P$ and $P \subseteq L$. By Lemma 7(ii), $P \in R d(L)$. Thus, $\vee_{L} P=\vee_{L^{\top}} P \geq x$ and $y \in P$. So, $y \models_{L^{\top}} x$ and $\Downarrow_{L} x \subseteq \Downarrow_{L^{\top}} x$ for each $x \in L$.

If $L$ itself is a (strongly) semicontinuous lattice, then $T$ is isolated in $L^{\top}$ and $L^{\top}$ is trivially a (strongly) semicontinuous lattice.
For a bc-dcpo $L$ without the biggest element, we see that $\vee_{L^{\top}} L=\top$. So, if $L$ is semicontinuous, then $\vee_{L^{\top}} \Downarrow_{L^{\top}} x \geq$ $\vee_{L^{\top}} \Downarrow_{L} x \geq x$ for each $x \in L$. It follows from

$$
\Downarrow_{L^{\top}} \top \supseteq \cup_{x \in L} \Downarrow_{L^{\top}} x \supseteq \cup_{x \in L} \Downarrow_{L} x
$$

that $\vee_{L^{\top}}\left(\Downarrow_{L^{\top}} T \cap \downarrow T\right)=\vee_{L^{\top}} \Downarrow_{L^{\top}} \vee^{\top} \geq \vee_{L^{\top}} \cup_{x \in L} \Downarrow_{L} x \geq$ $\vee_{L^{\top}} L=\mathrm{T}$. If $L$ is strongly semicontinuous, then for all $x \in$ $L, x \geq \vee_{L^{\top}}\left(\Downarrow_{L^{\top}} x \cap \downarrow x\right) \geq \vee_{L^{\top}}\left(\Downarrow_{L} x \cap \downarrow x\right) \geq x$ and $\vee_{L^{\top}}\left(\Downarrow_{L^{\top}} x \cap \downarrow x\right)=x$.

To sum up, if $L$ is a semicontinuous bc-domain, then $\forall y \in L^{\top}, y \leq \vee_{L^{\top}} \Downarrow_{L^{\top}} y$ and $L^{\top}$ is a semicontinuous lattice; if $L$ is a strongly semicontinuous bc-domain, then $\forall y \in L^{\top}$, $\vee_{L^{\top}}\left(\Downarrow_{L^{\top}} y \cap \downarrow y\right)=y$, and $L^{\top}$ is a strongly semicontinuous lattice.

Corollary 26. If $L$ is semicontinuous bc-domain, then $\forall x \in L$, $\Downarrow_{L^{\top}} x=\Downarrow_{L} x$.

Proof. By the proof of Proposition 25, we have that $\Downarrow_{L^{\top}} x \supseteq$ $\Downarrow_{L} x$ and $L^{\top}$ is a semicontinuous lattice. Then it follows from Propositions 12 and 15 and Lemma 7(ii) that $\Downarrow_{L^{\top}} x \subseteq \Downarrow_{L} x$. So, $\Downarrow_{L^{\top}} x=\Downarrow_{L} x$.

Whether the interpolation property holds or not in semicontinuous domains is still unknown. So, by Theorem 10 and Corollary 26, we immediately have the following.

Corollary 27. If $L$ is a (strongly) semicontinuous bc-domain, then $x \Leftarrow y$ implies the existence of a $z \in L$ such that $x \Leftarrow z \Leftarrow$ $y$.

Next we will show that (strongly) semicontinuous bcdomains also exhibit some strong types of interpolation properties.

Proposition 28. In a (strongly) semicontinuous bc-domain $L$,

(i) for all $x, y \in L$ with $x \leq y$, one has

$$
\left(S I^{\leq}\right) x \Leftarrow y \text { implies }(\exists z \in L) \quad(x \Leftarrow z \Leftarrow y, x \leq z) \text {; }
$$

(ii) for all $x, y \in L$ with $x<y$, one has

$$
\left(S I^{<}\right) x \Leftarrow y \text { implies }(\exists z \in L) \quad(x \Leftarrow z \Leftarrow y, x<z)
$$

Proof. (i) For any $x, y \in L, x \leq y$, and $x \Leftarrow y$, it follows from Corollary 27 that there exists a $z_{0} \in L$ such that $x \Leftarrow z_{0} \Leftarrow y$. Noticing that $\Downarrow y \in R d(L)$, by Proposition 6, there exists a $w \in R d(L)$ such that $x, z_{0} \leq w$. Since $L$ is a bc-dcpo, $x \vee z_{0}$ exists and $x \Leftarrow z_{0} \leq x \vee z_{0} \Leftarrow y$. Thus, $\left(S I^{\leq}\right)$holds.

(ii) Let $x, y \in L$ with $x<y$, and $x \Leftarrow y$. By Corollary 27 there exists a $z_{0} \in L$ such that $x \Leftarrow z_{0} \Leftarrow y$. Since $\Downarrow y \in$ $R d(L)$ and $y \leq \vee \Downarrow y$, there exists $z_{1} \in \Downarrow y$ such that $z_{1} \nless x$. Noticing that $z_{0}, z_{1} \in \Downarrow y \in R d(L)$, we see that $z_{0} \vee z_{1}$ exists. Set $z=z_{0} \vee z_{1}$; then, $x<z$. Therefore, $\left(S I^{<}\right)$holds. 


\section{Semi-FS Domains}

In this section, we introduce semi-FS domains which are counterparts of FS-domains posed by Jung [17] in the setting of strongly semicontinuous domains. It is proved that every strongly semicontinuous bc-domain, in particular every strongly semicontinuous lattice, is a semi-FS domain.

Definition 29 (see [16]). Let $L, M$ be dcpos. A function $f$ : $L \rightarrow M$ is said to preserve suprema of prime ideals if it is order-preserving and for any $P \in P I(L), f(\bigvee P)=\bigvee f(P)$.

Let $L, M$ be dcpos. We use $[L, M]$ to denote all orderpreserving functions from $L$ to $M$, use $[L \hookrightarrow M]$ to denote all the functions preserving suprema of prime ideals from $L$ to $M$ and use $[L \rightarrow M$ ] to denote all the Scott-continuous functions from $L$ to $M$. All of them are under the pointwise order. It is easy to see that $[L \rightarrow M] \subseteq[L \hookrightarrow M] \subseteq[L, M]$.

Proposition 30. Let $L, M$ be dcpos. Then $[L \hookrightarrow M]$ is a dcpo.

Proof. Let $\mathscr{G}$ be a directed subset of $[L \hookrightarrow M]$ and $f(x)=$ $\vee_{g \in \mathscr{G}} \mathcal{G}(x)$ for all $x \in L$. Then it is easy to see that $f$ is orderpreserving. For any prime ideal $P \in P I(L)$, we have

$$
\begin{aligned}
\vee f(P) & =\vee_{x \in P} f(x)=\vee_{x \in P} \vee_{g \in \mathscr{G}} \mathcal{g}(x)=\vee_{g \in \mathscr{G}} \vee g(P) \\
& =\vee_{g \in \mathscr{G}} \mathcal{G}(\vee P)=f(\vee P) .
\end{aligned}
$$

Thus, $f \in[L \hookrightarrow M]$, showing that $[L \hookrightarrow M]$ is a dcpo.

Definition 31. Let $L$ be a dcpo. If $\mathscr{D} \subseteq[L, L]$ is directed and $\sup _{\delta \in \mathscr{D}} \delta=I d_{L}$, then we say that $\mathscr{D}$ is an approximate identity for $L$.

Proposition 32. Let $L$ be a dcpo. If $L$ has an approximate identity $\mathscr{D} \subseteq[L \hookrightarrow L]$ such that $\delta(x) \Leftarrow x$ for all $\delta \in \mathscr{D}$ and for all $x \in L$, then $L$ is a strongly semicontinuous domain.

Proof. For any $x \in L$, let $A=\{\delta(x): \delta \in \mathscr{D}\}$. Then $A \subseteq \Downarrow x \cap \downarrow x$. Note that $\mathscr{D}$ is directed and $x \in \downarrow(\vee A)=A \uparrow \downarrow$. By the Proposition 16 and Definition 17, it follows that $L$ is a strongly semicontinuous domain.

Definition 33 (see [17]). Let $L$ be a dcpo. A function $\delta: L \rightarrow$ $L$ on $L$ is finitely separating if there is a finite set $F_{\delta}$ such that, for each $x \in L$, there exists $y \in F_{\delta}$ such that $\delta(x) \leq y \leq x$.

A dcpo $L$ is called a semi-FS domain if there is an approximate identity $\mathscr{D} \subseteq[L \hookrightarrow L]$ consisting of finitely separating functions.

For an FS-domain $L$, there is an approximate identity $\mathscr{D} \subseteq$ $[L \rightarrow L] \subseteq[L \hookrightarrow L]$ for $L$ consisting of finitely separating functions. So, an FS-domain is a semi-FS domain.

Proposition 34. Let $L$ be a dcpo. If $\delta \in[L \hookrightarrow L]$ is finitely separating, then, for all $x \in L, \delta(x) \Leftarrow x$. Thus a semi-FS domain is a strongly semicontinuous domain.

Proof. Suppose that $x \in L$ and $P \in P I(L)$ with $x \leq \vee P$. Since $\delta$ is finitely separating, there exists a finite set $F_{\delta}$ such that for

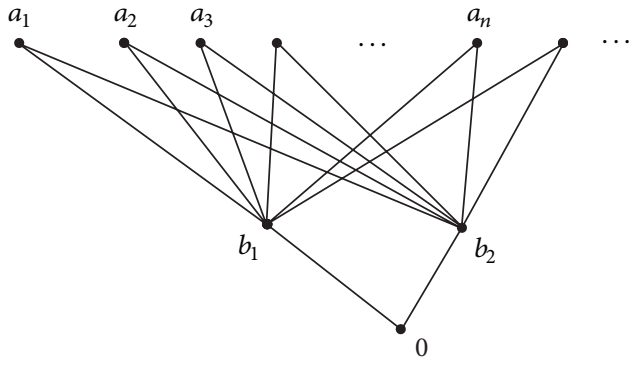

FIGURE 1: $L$ is strongly semicontinuous but not a semi-FS domain.

each $d \in P$ there exists $y_{d} \in F_{\delta}$ with $\delta(d) \leq y_{d} \leq d$. Let $F_{\delta}^{\prime}=\left\{y_{d} \in F_{\delta}: d \in P\right\}$, a nonempty finite subset of $F_{\delta}$. Then for each $y \in F_{\delta}^{\prime}$, we can get $d_{y} \in P$ such that $\delta\left(d_{y}\right) \leq y \leq d_{y}$. As $P$ is a prime ideal, there exists $d_{0} \in P$ such that $y \leq d_{y} \leq d_{0}$ for all $y \in F_{\delta}^{\prime}$. Hence for all $d \in P, \delta(d) \leq y_{d} \leq d_{0}$ and $\delta(x) \leq$ $\delta(\vee P)=\vee_{d \in P} \delta(d) \leq d_{0}$. Therefore, $\delta(x) \in P$. It follows from Proposition 12 that $\delta(x) \Leftarrow x$.

By Proposition 32 we see that $L$ is a strongly semicontinuous domain.

The next example gives a strongly semicontinuous domain which is not a semi-FS domain, showing that the reverse of Proposition 34 is not true.

Example 35. Let $L=\left\{0, b_{1}, b_{2}\right\} \cup\left\{a_{j}: j=1,2, \ldots\right\}$ be the domain showing in Figure 1, where $0<b_{i}<a_{j}$ for $i=1,2$ and $j=1,2, \ldots$. It is clear that $L$ is an $L$-domain but not compact in the Lawson topology. By [20, Corollary 2.2], we immediately see that $L$ is not an FS-domain. Note that, in $L$, every directed set is finite. So, an order-preserving function from $L$ to $L$ preserves directed sups in $L$. And $[L \rightarrow L] \subseteq$ $[L \hookrightarrow L] \subseteq[L, L]=[L \rightarrow L]$. So, $[L \rightarrow L]=[L \hookrightarrow L]$. Since $L$ is not an FS-domain, $L$ is not a semi-FS domain either.

Proposition 36. Every strongly semicontinuous bc-domain is a semi-FS domain.

Proof. Let $L$ be a strongly semicontinuous bc-domain. For each $x \in L, S \in \mathscr{P}_{\text {fin }}(L)$, define $\delta_{S}: L \rightarrow L$ by $\delta_{S}(x)=$ $\vee\{y \in S \cap \downarrow x: y \Leftarrow x\}$. If $\{y \in S \cap \downarrow x: y \Leftarrow x\}=\emptyset$, then $\delta_{S}(x)=\perp$, the least element of $L$. So, $\delta_{S}(x)$ is well-defined. It is easy to see that $\delta_{S}$ is order-preserving with $\delta_{S}(x) \leq x$ for all $x \in L$. Next we show that $\delta_{S}$ preserves suprema of prime ideals. For each $P \in P I(L)$, it suffices to show that $\delta_{S}(\vee P)=\delta_{S}(k) \leq \vee_{p \in P} \delta_{S}(p)$, where $k=\vee P$. If $\{y \in S \cap$ $\downarrow k: y \Leftarrow k\}=\emptyset$, then, by the definition of $\delta_{S}$, we see that $\delta_{S}(k)=\perp \leq \vee_{p \in P} \delta_{S}(p)$. Let $\left\{y_{1}, \ldots, y_{l}\right\}=\{y \in S \cap \downarrow k: y \Leftarrow$ $k\} \neq \emptyset$, then $m=\vee_{i=1}^{l} y_{i}$ exists in $L$. By the definition of $\delta_{S}$, $\delta_{S}(k)=m \leq k$. Since $y_{i} \Leftarrow k$ for $i=1,2, \ldots, l$, we have that $m \Leftarrow k$. By $\left(\mathrm{SI}^{\leq}\right)$in Proposition 28, there is a $m^{*} \geq m$ such that $m \Leftarrow m^{*} \Leftarrow k$. It follows from $m^{*} \Leftarrow k=\vee P$ that $m, m^{*} \in P$. Noticing that $m \leq m^{*}$ and $y_{i} \leq m \Leftarrow m^{*} \in P$, we have $\left\{y_{1}, \ldots, y_{l}\right\} \subseteq\left\{y \in S \cap \downarrow m^{*}: y \Leftarrow m^{*}\right\}$ which yields that $\vee_{p \in P} \delta_{S}(p) \geq \delta_{S}\left(m^{*}\right) \geq \delta_{S}(k)$. So, $\delta_{S}$ preserves suprema of prime ideals, and $\delta \in[L \hookrightarrow L]$. 
Suppose that $S, T \in \mathscr{P}_{\text {fin }}(L)$ with $S \subseteq T$. It is easy to see that $\delta_{S} \leq \delta_{T}$ and $\mathscr{D}=\left\{\delta_{S}\right\}_{S \in \mathscr{P}_{\text {fin }}(L)}$ is directed. Let $F_{\delta_{S}}=\left\{\delta_{S}(x): x \in L\right\}$. Then $F_{\delta_{S}}$ is finite by the finiteness of $S$. So, $\delta_{S}$ is a finitely separating function. Since $L$ is strongly semicontinuous, for each $x \in L$ and $\delta_{S} \in \mathscr{D}, \delta_{S}(x)=\vee\{y \in$ $S \cap \downarrow x: y \Leftarrow x\} \leq x$ and

$$
\begin{aligned}
\vee_{\delta_{S} \in \mathscr{D}} \delta_{S}(x) & =\vee_{\delta_{S} \in \mathscr{D}}(\vee\{y \in S \cap \downarrow x: y \Leftarrow x\}) \\
& \geq \vee_{z \Leftarrow x}(\vee\{y \in\{z\} \cap \downarrow x: y \Leftarrow x\}) \\
& =\vee(\Downarrow x \cap \downarrow x)=x .
\end{aligned}
$$

Therefore, $\mathscr{D}=\left\{\delta_{S}\right\}_{S \in \mathscr{P}_{\text {fin }}(L)} \subseteq[L \hookrightarrow L]$ is an approximate identity for $L$ consisting of finitely separating functions, and $L$ is a semi-FS domain.

Corollary 37. Every strongly semicontinuous lattice is a semiFS domain.

\section{Conflict of Interests}

The authors declare that there is no conflict of interests regarding the publication of this paper.

\section{Acknowledgments}

The authors are grateful to the anonymous reviewers for their valuable comments and helpful suggestions. This work is supported by the NSF of China $(61103018,61300153)$ and Open Fund of the State Key Laboratory of Beijing University (no. SKLSDE-2011KF-08).

\section{References}

[1] D. S. Scott, "Continuous lattices," in Toposes, Algebraic Geometry and Logic, vol. 274 of Lecture Notes in Mathematics, pp. 97-136, Springer, Berlin, Germany, 1972.

[2] D. S. Scott, "Domains for denotational semantics", in Automata, Languages and Programming, vol. 140 of Lecture Notes in Computer Science, pp. 577-613, Springer, Berlin, Germany, 1982.

[3] Y. Chen and H. Wu, "Domain semantics of possibility computations," Information Sciences, vol. 178, no. 12, pp. 2661-2679, 2008.

[4] G. Gierz, K. H. Hofmann, K. Keimel, J. D. Lawson, M. Mislove, and D. S. Scott, Continuous Lattices and Domains, Cambridge University Press, Cambridge, UK, 2003.

[5] G. Zhang, Logic of Domains. Progress in Theoretical Computer Science, Birkhäauser, Boston, Mass, USA, 1991.

[6] D. Spreen, L. Xu, and X. Mao, "Information systems revisitedthe general continuous case," Theoretical Computer Science, vol. 405, no. 1-2, pp. 176-187, 2008.

[7] G. Gierz, J. D. Lawson, and A. Stralka, "Quasicontinuous posets," Houston Journal of Mathematics, vol. 9, no. 2, pp. 191208, 1983.

[8] X. Mao and L. Xu, "Quasicontinuity of posets via Scott topology and sobrification," Order, vol. 23, no. 4, pp. 359-369, 2006.

[9] J. B. Wright, E. G. Wagner, and J. W. Thatcher, "A uniform approach to inductive posets and inductive closure," Theoretical Computer Science, vol. 7, no. 1, pp. 57-77, 1978.
[10] M. Erné, " $Z$-Continuous Posets and Their Topological Manifestation," Applied Categorical Structures, vol. 7, no. 1-2, pp. 31-70, 1999.

[11] Y. Rav, "Semiprime ideals in general lattices," Journal of Pure and Applied Algebra, vol. 56, no. 2, pp. 105-118, 1989.

[12] D. Zhao, "Semicontinuous lattices," Algebra Universalis, vol. 37, no. 4, pp. 458-476, 1997.

[13] Q. G. Li and X. H. Wu, "Generalizations and Cartesian closed subcategories of semicontinuous lattices," Acta Mathematica Scientia B, vol. 29, no. 5, pp. 1366-1374, 2009.

[14] H. Y. Bi and X. Q. Xu, "Semi-Scott topology and semiLawson topology on semicontinuous lattices," Fuzzy Systems and Mathematics, vol. 22, no. 2, pp. 75-81, 2008 (Chinese).

[15] G. H. Jiang and W. X. Shi, "Characterizations of distributive lattices and semicontinuous lattices," Bulletin of the Korean Mathematical Society, vol. 47, no. 3, pp. 633-643, 2010.

[16] G. L. Li, Some generalizations and applications of domain theory [Ph.D. thesis], Yangzhou University, Yangzhou, China, 2012.

[17] A. Jung, "Cartesian Closed Categories of Domains," CWI Tracks 66, Centrum voor Wiskunde en Informatica, Amsterdam, The Netherlands, 1989.

[18] Q. Y. He and L. S. Xu, "Strongly semicontinuous lattices," Bulletin of the Korean Mathematical Society. Submitted.

[19] X. H. Wu and Q. G. Li, "Quasi-semicontinuous lattices and meet-semicontinuous lattices," Fuzzy Systems and Mathematics, vol. 22, no. 6, pp. 11-16, 2008 (Chinese).

[20] J. H. Liang and K. Keimel, "Compact continuous L-domains," Computers \& Mathematics with Applications, vol. 38, no. 1, pp. 81-89, 1999. 


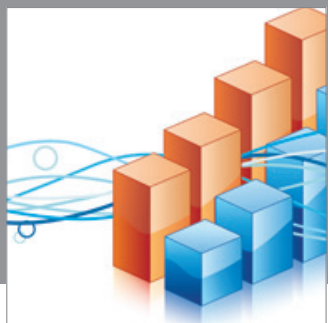

Advances in

Operations Research

mansans

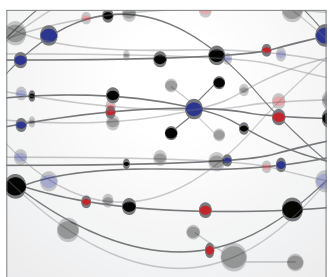

The Scientific World Journal
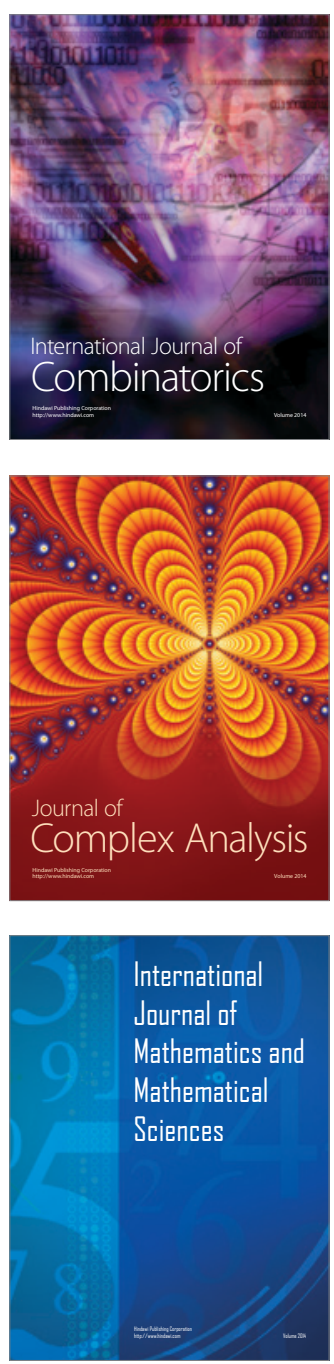
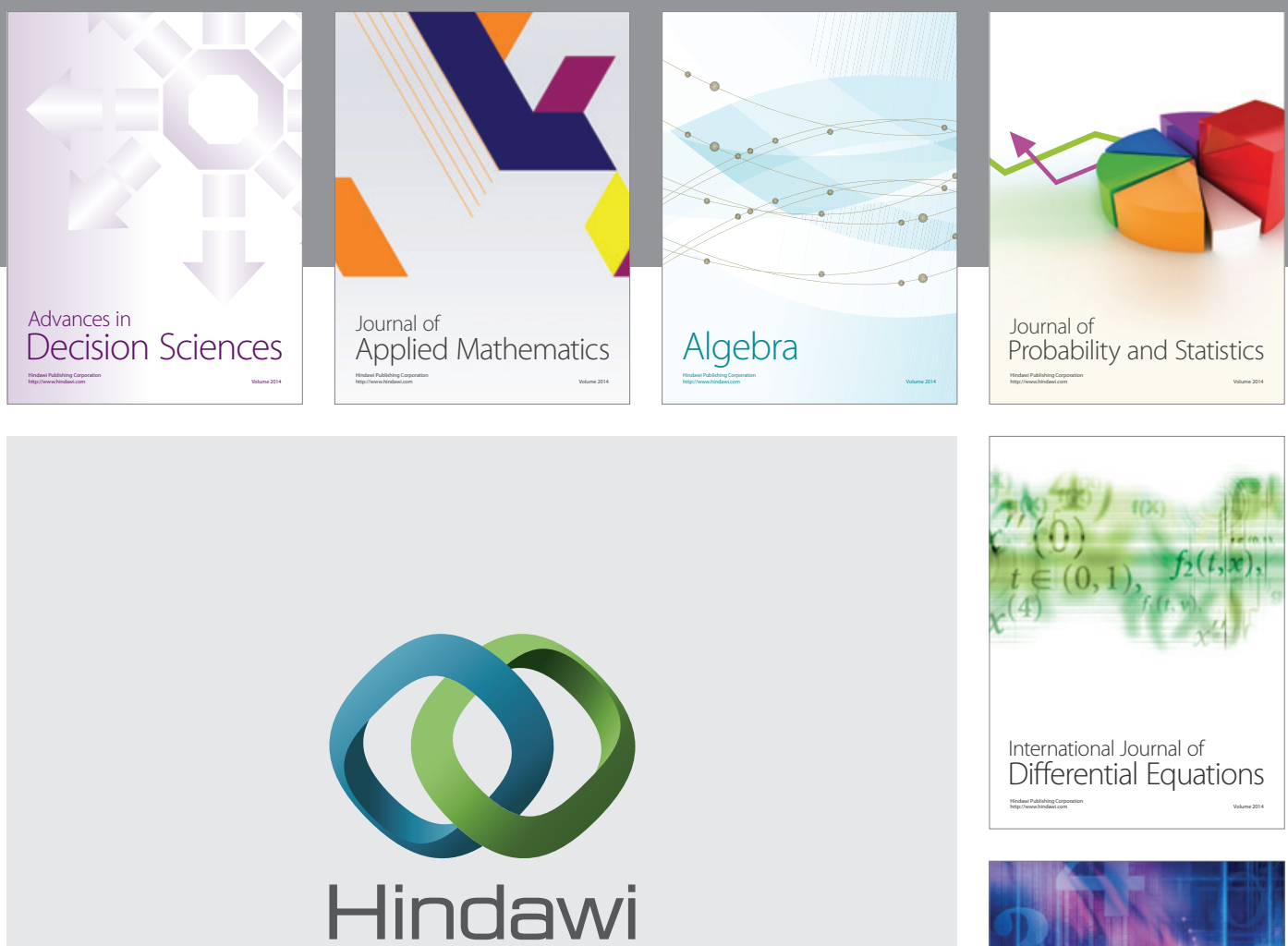

Submit your manuscripts at http://www.hindawi.com
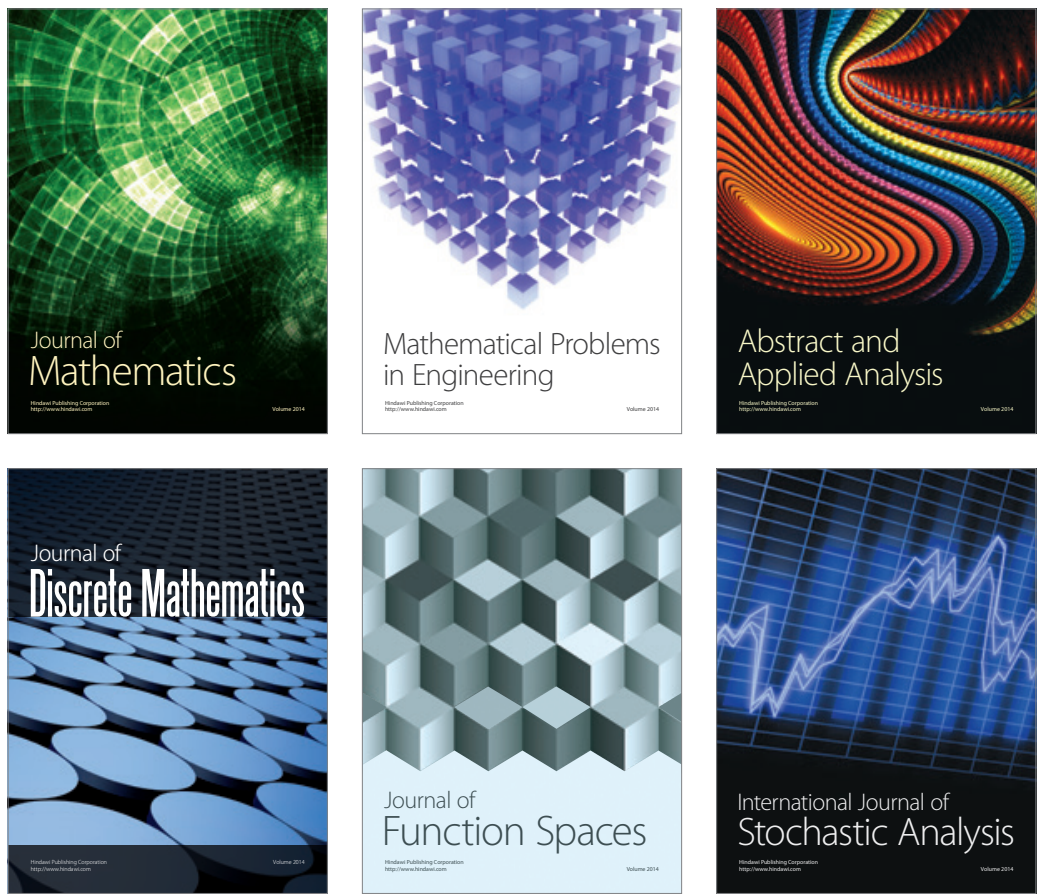

Journal of

Function Spaces

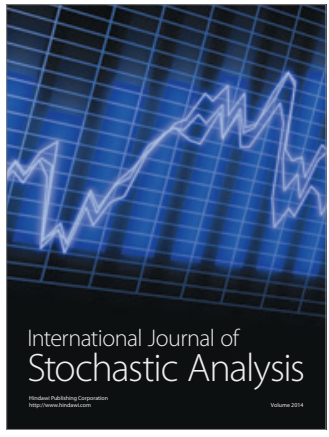

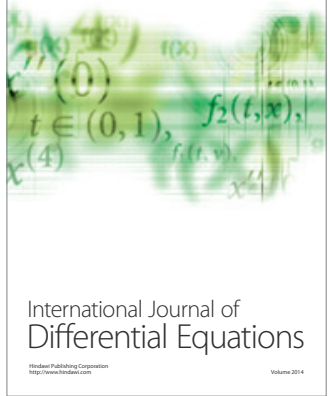
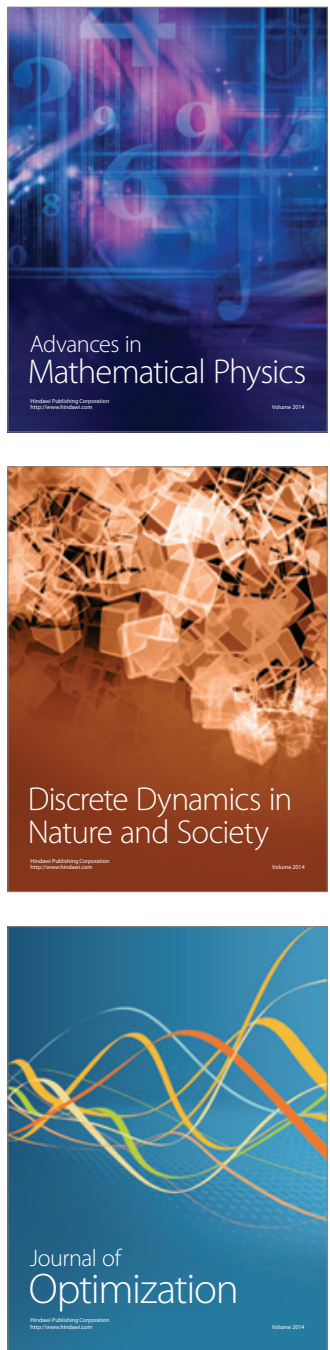